\title{
Ultra-Localization of Foxp3 ${ }^{+}$T Cells within Renal Allografts Shows Infiltration of Tubules Mimicking Rejection
}

\author{
Kathryn Brown, Victoria Moxham, Julieta Karegli, \\ Richard Phillips, Steven H. Sacks, and \\ Wilson Wong \\ From the Department of Nephrology and Transplantation, \\ King's College London School of Medicine at Guy's, King's and \\ St Thomas' Hospitals, London, United Kingdom
}

Kidney transplant recipients are monitored for rejection by measurement of serum creatinine and graft biopsies. Biopsy samples are evaluated according to the Banff classification, which states that infiltration of tubules by mononuclear cells is an indicator of acute rejection. However, regulatory $T$ cells play a crucial role in the overall immune response and are also present within transplanted tissue. We hypothesize that infiltration of mononuclear cells within kidney grafts is not always associated with rejection, especially if a high proportion of this infiltrate is regulatory $T$ cells. Using a life-sustaining mouse kidney transplant model, we found that mononuclear cell tubular infiltration can occur in both rejecting and tolerant grafts. However, tolerant kidney grafts demonstrated a higher and sustained level of Foxp $3^{+}$ regulatory cells. Importantly, a significant proportion of these cells were found within tubules. In cases in which graft function was normal, these cells were not harmful to the kidney and could be said to be mimicking, rather than causing, rejection. (Am J Pathol 2007, 171:1915-1922; DOI: 10.2353/ajpath.2007.070396)

Kidney transplantation is the best form of treatment for end-stage renal failure. Despite a high success rate with graft survival of more than $90 \%$ in the 1 st year, acute rejection remains the principal cause of graft loss within the 1st year of transplantation and contributes to chronic damage. Although serum creatinine is a good measurement of renal function, rejection can only be reliably diagnosed by histological analysis of biopsy samples. ${ }^{1}$

The Banff classification ${ }^{2}$ is now universally used for histological assessment of rejection in human renal trans- plantation. Type I-tubulointerstitial rejection without arteritis - can only be diagnosed when "interstitial infiltration and foci of tubulitis" are seen. Interstitial infiltration alone, without tubulitis (defined as the presence of mononuclear cells within tubules), is not diagnostic of rejection. This is in sharp contrast to the diagnosis of rejection after cardiac transplantation in which rejection can be based on mononuclear cell infiltration alone. ${ }^{3}$

Regulatory T cells (Tregs) have been investigated extensively in human and animal models of transplantation tolerance (reviewed in Ref. ${ }^{4}$ ). Although there is no single surface marker that defines $T$ cells with regulatory properties, they are highly enriched in the $\mathrm{CD} 4^{+} \mathrm{CD} 25^{+} \mathrm{T}$-cell population. In mice, expression of the intracellular transcription factor Foxp3 is exclusively associated with T cells possessing regulatory activity. ${ }^{5-7}$ After transplantation, Tregs have been found in secondary lymphoid tissues such as spleen ${ }^{8}$ and lymph nodes ${ }^{9}$ and within the transplanted graft. ${ }^{10}$

It has been suggested that during a rejection response, effector lymphocytes and Tregs are formed and that it is the balance between these two populations that determine graft outcome. ${ }^{11}$ Higher urinary FOXP3 mRNA levels (presumed to be derived from graft infiltrating leukocytes leaking into the urine) in patients undergoing rejection are associated with better outcome and longterm graft survival. ${ }^{12}$

We hypothesize that interstitial infiltration of mononuclear immune cells in renal allografts, even when foci of tubulitis is seen, does not necessarily equate to rejection, particularly when the ratio of Foxp3 ${ }^{+}$cells with regulatory properties to other T cells is high.

To address this, we used a life-sustaining mouse model of renal transplantation in which spontaneous acceptance occurs in certain strain combinations without immunosuppression. Graft function can be monitored to confirm the absence of "clinical rejection" as defined by a rise in blood urea nitrogen (BUN). Transplanted allografts were exam-

\footnotetext{
Supported by a grant from Genzyme Renal Innovations Program. Accepted for publication August 30, 2007

Address reprint requests to Dr. Wilson Wong, Department of Nephrology and Transplantation, 5th Floor, Thomas Guy House, Guy's Hospital, London SE1 9RT, UK. E-mail: wilson.wong@kcl.ac.uk.
} 
ined for histological signs of rejection and for the presence of infiltrating Foxp ${ }^{+}$cells to compare recipients with normal graft function with those rejecting their grafts. In addition, heterotopic cardiac transplants were also performed for further comparison between tolerance and rejection within the same strain combination.

\section{Materials and Methods}

\section{Animals}

Female donor BALB/C $\left(\mathrm{H}-2^{d}\right)$, DBA/2 $\left(\mathrm{H}-2^{d}\right)$ or CBA.Ca $\left(\mathrm{H}-2^{\mathrm{k}}\right)$, and recipient $\mathrm{C} 57 \mathrm{BL} / 6\left(\mathrm{H}-2^{\mathrm{b}}\right)$ mice were purchased from Harlan UK Ltd. (Oxon, UK), kept in specific pathogen-free animal facilities, and used in accordance with the Animals (Scientific Procedures) Act 1986.

\section{Organ Transplantation}

Renal and cardiac transplantation were performed as described previously. ${ }^{13,14}$ For renal allograft survival experiments, the recipient left native kidney was removed at the time of transplantation. The remaining right native kidney was removed 1 week after transplantation, leaving the donor graft life sustaining. Before and at regular intervals after the second native nephrectomy, blood samples were obtained through tail vein bleeding, and measurements of BUN were performed to monitor graft function. Skin transplantation was performed as described previously. ${ }^{15}$

\section{Depletion of $\mathrm{CD}^{2} 5^{+} \mathrm{T}$ Cells}

In some experiments, organ recipients were given $4 \times$ $250 \mu \mathrm{g}$ of the depleting anti-CD25 antibody (clone PC61; Bioexpress, West Lebanon, NH) on days $0,1,2$, and 3. The first dose was given intravenously, with subsequent administrations given intraperitoneally.

\section{Blood Urea Nitrogen Measurement}

Serum BUN was measured using Infinity Urea (Clindia Benelux, BV, Leusden, The Netherlands) according to manufacturer's instruction.

\section{Tissue Harvesting}

At various time points after transplantation, grafted organs and spleens from C57BL/6 recipients were harvested in optimal cutting temperature compound (Raymond A. Lamb Ltd., Eastbourne, UK) for frozen sections and $4 \%$ formaldehyde for processing into paraffin blocks.

\section{Histology}

Periodic acid Schiff staining was performed on 2- $\mu \mathrm{m}$ thick paraffin sections as previously described. ${ }^{16}$

\section{Immunohistochemistry}

Frozen tissue samples were cut into $5-\mu \mathrm{m}$ sections, transferred onto slides, and left to dry for 1 hour. They were then fixed in acetone. Endogenous peroxidase activity within the tissue was quenched with $3 \% \mathrm{H}_{2} \mathrm{O}_{2}$ in PBS (Oxoid Ltd., Basingstoke, UK), and avidin/biotin with a blocking kit (Vector Laboratories, Burlingame, CA) used according to manufacturer's instructions. The primary antibody was added for 1 hour, and the secondary (biotinylated) for 30 minutes. After adding streptavidin-horseradish peroxidase conjugate (Pharmingen, San Diego, CA), color was developed using Vector NovaRed Substrate kit (Vector Laboratories), and the sections were counterstained with Mayer's Hemalum (Raymond A Lamb Ltd.), followed by dehydration and mounting in DPX mountant (BDH, Leicestershire, UK).

Foxp3 was identified using a biotinylated antibody (clone FJK-16s; eBioscience, San Diego, CA) incubated overnight; whereas anti-CD4 (clone H129.19), anti-CD8 (clone 53-6.7), anti-CD68 (clone FA-11), and anti-CD19 (clone 1D3) were purified primary rat anti-mouse antibodies detected by a biotinylated goat anti-rat [all Pharmingen except anti-CD68 (Serotec, Oxford, UK)]. A rat IgG2a, clone R35-95 (Pharmingen) was used as an isotype control.

Paraffin sections were cut at $2 \mu \mathrm{m}$ and dried at $60^{\circ} \mathrm{C}$ overnight before deparaffinizing in xylene. Antigen retrieval was performed by boiling slides in sodium citrate buffer before staining as above.

Positive cells were counted in 20 random high-power fields (HPFs; $\times 400$ ) of each sample by two independent observers blinded to the experimental conditions. Results were expressed as means \pm SEM.

\section{Flow Cytometry}

Peripheral blood was stained with anti-Foxp3 antibody (clone FJK-16s) after lysing with FACSlyse (Pharmingen). Cells were acquired using a FACScan flow cytometer (Becton Dickinson, Oxford, UK) and analyzed using Cellquest software version V3.3 (Becton Dickinson).

\section{Statistics}

Unpaired two-tailed Student's $t$-tests were used for all results except survival, which was tested by the log rank sum test. Correlation of BUN with Foxp $3^{+}$T-cell infiltration was tested with linear regression analysis.

\section{Results}

\section{Rejection and Spontaneous Acceptance of Mouse Kidney Allografts from Different Strains of Allogeneic Donors Both with $\mathrm{H}-2^{d}$ Haplotype}

When BALB/c kidney allografts were transplanted into C57BL/6 recipients, the majority were rejected acutely with a sharp rise in BUN after the second native nephrec- 

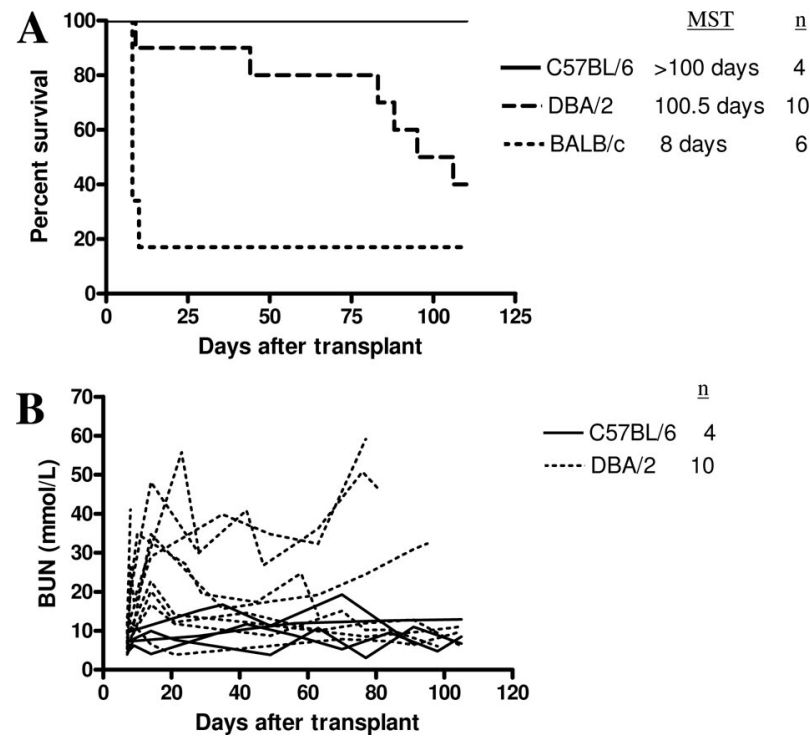

Figure 1. A: Survival of $\mathrm{C} 57 \mathrm{BL} / 6, \mathrm{DBA} / 2$, and $\mathrm{BALB} / \mathrm{c}$ kidney grafts in $\mathrm{C} 57 \mathrm{BL} / 6$ recipients. Although syngeneic grafts survived indefinitely, most $\mathrm{BALB} / \mathrm{c}$ allografts were rejected. $\mathrm{DBA} / 2$ grafts, which are major histocompatibility complex identical to BALB/c, displayed prolonged survival with some mice accepting their allografts indefinitely with relatively normal BUN. B: Renal function as measured by BUN after transplantation of C57BL/ 6 or DBA/2 kidney grafts.

tomy (median survival time $=8$ days, $n=6$; Figure 1). By contrast, kidney grafts from DBA/2 mice, which are major histocompatibility complex identical to BALB/C, displayed prolonged survival in C57BL/6 recipients (median survival time $=100.5$ days, $n=10, P=0.0474$; Figure 1), in concordance with published data. ${ }^{17}$ Survival of challenge skin grafts from DBA/2 mice was prolonged in C57BL/6 recipients of DBA/2 kidneys (graft survival of 18 , 19 , and $>100$ days). By contrast, all third party CBA skin grafts were rejected acutely (graft survival of 11,12 , and 12 days) $(P=0.03)$. All DBA/2 heart allografts were rejected acutely by $\mathrm{C} 57 \mathrm{BL} / 6$ recipients (median survival time $=13$ days, $n=3$ ).

\section{Presence of Regulatory T Cells within Both Rejecting and Nonrejecting Renal Allografts}

Despite the relatively normal graft function in recipients of DBA/2 kidney grafts, cellular infiltrates could be seen with foci of tubulitis, satisfying the Banff criteria for acute rejection. This was observed at 8 and 180 days after transplantation (Figure 2, A and B). This led us to investigate whether some of the graft-infiltrating cells were Tregs and therefore beneficial rather then detrimental to the transplanted graft. At early time points, eg, day 4 after transplantation, Foxp3 ${ }^{+}$cells could be seen in DBA/2 and BALB/c kidney allografts, and numbers were slightly, but not significantly, higher in DBA/2 grafts (15.25 \pm 5.18 and $7.56 \pm 2.14$ per HPF, respectively) (Figures $3 \mathrm{~A}$ and 2D). Importantly, at later time points (14 to 80 days after transplantation), recipients of DBA/2 kidney transplants that had retained their grafts with good function maintained high levels of Foxp3 expression (20.92 \pm 0.96 per HPF at 14 days and $25.27 \pm 2.9$ per HPF after 45 days).
By day 8, BALB/c donor kidneys were severely damaged by the rejection process, with much of the tissue infarcted. Although immunohistochemical staining was performed, accurate analysis of the sections could not be performed, and only one of four samples could be evaluated and plotted in Figure 3 on day 8 for BALB/C donor kidneys. Therefore, we have not attempted to compare data for BALB/c donor kidneys with DBA/2 donor kidneys at this particular time point. Foxp ${ }^{+}$cells were rare in syngeneic kidney and DBA/2 and syngeneic heart grafts $(1.11 \pm 0.45,1.25 \pm 0.96$ and $0.17 \pm 0.08$ per HPF at day 4 , respectively) (Figures $3 \mathrm{~A}$ and $2 \mathrm{E}$ ).

A similar pattern was found when total $\mathrm{CD}^{+}{ }^{+} \mathrm{T}$ cells were analyzed. Low numbers were found in syngeneic kidney and in DBA/2 and syngeneic heart grafts (23.66 \pm $0.4,24.15 \pm 4.68$, and $10.43 \pm 1.24$ per HPF at day 4 , respectively) (Figures $3 \mathrm{~B}$ and $2 \mathrm{~F}$ ). Similar numbers were found in DBA/2 and BALB/c kidney allografts (72.78 \pm 11.32 and $67.62 \pm 7.84$ per HPF at day 4 , respectively) (Figures $3 \mathrm{~B}$ and $2 \mathrm{G}$ ). The ratio of Foxp3 ${ }^{+}$cells to total CD4-positive cells was therefore higher in DBA/2 kidney allografts compared with BALB/c (1:5 compared with 1:9) and much lower in the other groups (1:25 in syngeneic kidney grafts and 1:20 in DBA/2 heart grafts) on day 4 . In this model, analysis was only possible at this time point because the number of Foxp3 ${ }^{+}$cells were low at early time points, whereas at later time points, most of the BALB/c kidneys were completely destroyed by the rejection process, making immunohistochemical analysis impossible.

Staining for CD8, CD68 (a macrophage marker), and CD19 (a B-cell marker) at day 4 after transplant revealed no differences between tolerant $\mathrm{DBA} / 2$ and rejecting BALB/c kidney allografts. There were $59.4 \pm 9.7$ and $97.3 \pm 17.4 \mathrm{CD}^{+}$cells per HPF; $10.5 \pm 0.8$ and $14.5 \pm$ $1.6 \mathrm{CD} 8^{+}$cells per HPF; $6.3 \pm 1.4$ and $6.9 \pm 1.5 \mathrm{CD}^{+}{ }^{+}$ cells per HPF in DBA/2 and BALB/c kidney allografts, respectively.

Although a high proportion of DBA/2 kidney grafts were accepted by C57BL/6 recipients without immunosuppression, some did show a slow decline in graft function, which eventually lead to death of the recipient from graft failure. Interestingly, in this series of experiments, three DBA/2 kidney recipients had deranged graft function at the time of sacrifice, and all three of these grafts contained very few $\mathrm{Foxp}^{+}$cells. When the number of graft-infiltrating Foxp ${ }^{+}$cells was plotted against BUN at the time of sacrifice, a strong correlation was found. Animals whose graft contained low numbers of Foxp3 ${ }^{+}$ cells had much higher BUNs than those with more infiltrating Foxp ${ }^{+}$cells $(P=0.002)$, suggesting a link between graft function and regulatory $T$ cells (Figure 4). Periodic acid Schiff sections of grafts with good longterm function showed well-preserved tubular architecture but numerous clusters of heavy mononuclear cellular infiltrate (Figure $2 \mathrm{H}$ ), with foxp3 ${ }^{+}$cells among them (Figure 2l). By contrast, grafts with poor long-term function did not have these areas of mononuclear cellular infiltrate but showed tubular atrophy, interstitial expansion, glomerular sclerosis, and hyline cast, typical of chronic allograft nephropathy (Figure 2J). 

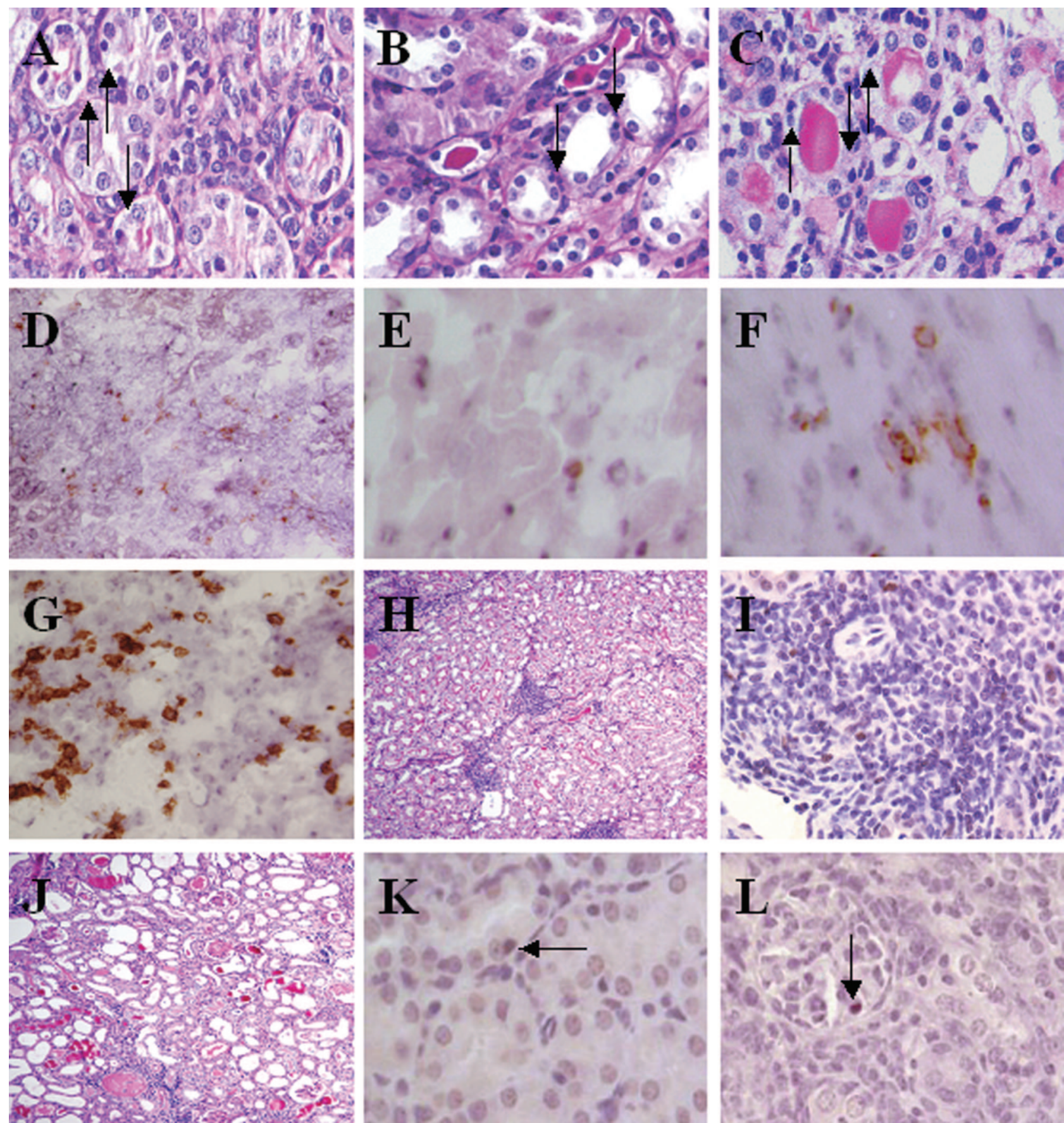

Figure 2. Periodic acid Schiff-stained sections showing mononuclear infiltration of tubules in DBA/2 kidney allografts 8 days (A) and 180 days (B) after transplant C: BALB/c kidney allograft day 8 after transplant. D: Foxp3 staining in DBA/2 kidney graft. E: Foxp3 staining in DBA/2 heart graft. F: CD4 staining in DBA/2 heart graft. G: CD4 staining in DBA/2 kidney graft. H: Representative example of Periodic acid Schiff section of long-term DBA/2 kidney allograft with low BUN and high Foxp $3^{+}$cell count, showing clusters of mononuclear infiltrate but normal tubular architecture. In this particular example, the kidney allograft was harvested 185 days after transplantation, BUN was $10.64 \mathrm{mmol} / \mathrm{L}$, and Foxp $3^{+}$cell count was 18.56 per HPF. I: Foxp $3^{+}$cells can be seen within clusters of mononuclear infiltrate in a DBA/2 kidney allograft with normal function harvested 45 days after transplantation. J: Representative example of Periodic acid Schiff section of long-term DBA/2 kidney allograft with poor graft function and low Foxp3 ${ }^{+}$cell count, showing features of chronic allograft nephropathy. In this particular example, even though the kidney allograft was harvested 88 days after transplantation, earlier than the examples shown in $\mathbf{B}$ and $\mathbf{H}$, the BUN was higher at 56.91 mmol/L and Foxp3 $3^{+}$cell count was lower at 1.14 per HPF. K: Foxp3 staining on paraffin sections show Foxp $3^{+}$cells present within a tubule. L: Foxp3 staining on paraffin sections show Foxp $3^{+}$cells present within a glomerulus. Original magnifications: $\times 400(\mathbf{A}, \mathbf{C}, \mathbf{D}, \mathbf{E}, \mathbf{F}, \mathbf{G}, \mathbf{I}, \mathbf{K}$, and $\mathbf{L}), \times 250(\mathbf{B})$, and $\times 100(\mathbf{H}$ and $\mathbf{J})$.

\section{Localization of Foxp3 ${ }^{+}$Cells within Allografts} Shows Foci of Tubulitis, Mimicking Rejection

Although Foxp3 staining on frozen sections was reliable and allowed comparisons with CD4 staining, the archi- tecture of the kidney was not well preserved during staining. Therefore Foxp3 immunohistochemical staining was performed on paraffin-fixed sections of kidney allografts to better visualize the architecture of the kidney and allow the localization of $\mathrm{Foxp3}^{+}$cells within these organs. 
A

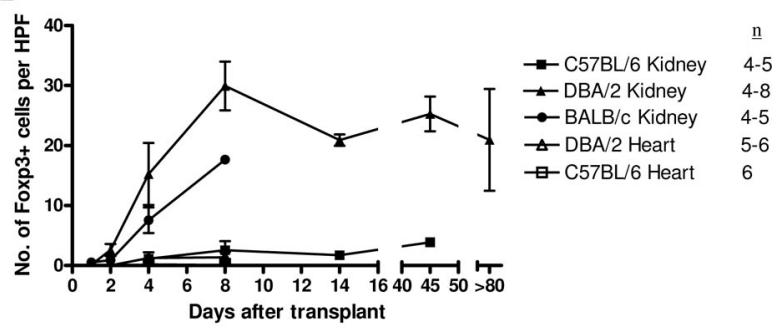

B

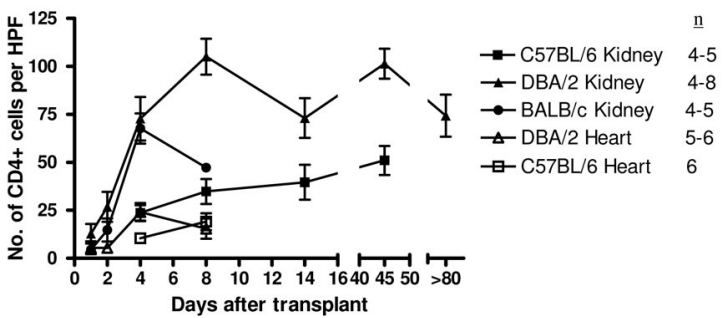

Figure 3. A: Foxp3 staining in syngeneic and allogeneic kidney and heart transplants shows increased levels in allogeneic compared with syngeneic kidney grafts and all heart grafts. B: A similar pattern is seen in overall numbers of $\mathrm{CD}^{+} \mathrm{T}$ cells.

Figure 5 shows that as with the mononuclear infiltrate into the kidney, most Foxp3 ${ }^{+}$cells were found within the interstitium, but a significant proportion of these cells were seen infiltrating tubules, mimicking tubulitis (Figures 5 and $2 \mathrm{~K})$. Very rarely, Foxp3 ${ }^{+}$cells were observed within glomeruli (Figures 5 and 2L). Tubule-infiltrating Foxp3 ${ }^{+}$ cells were seen in all groups (DBA/2, BALB/c, and C57BL/6 kidney grafts) at all time points tested. Interestingly, there was no significant difference between the number of Foxp3 ${ }^{+}$cells infiltrating the tubules of tolerant $\mathrm{DBA} / 2$ kidneys and rejecting BALB/c kidneys. Paradoxically, in this set of experiments, higher numbers (although not statistically significant) of Foxp ${ }^{+}$cells were found in the interstitium and infiltrating tubules in BALB/C grafts compared with DBA/2 grafts at day 8 . However, this must be interpreted with caution because most BALB/c grafts were intact enough to render immunohistochemistry possible $(n=2)$. Therefore, the results at this

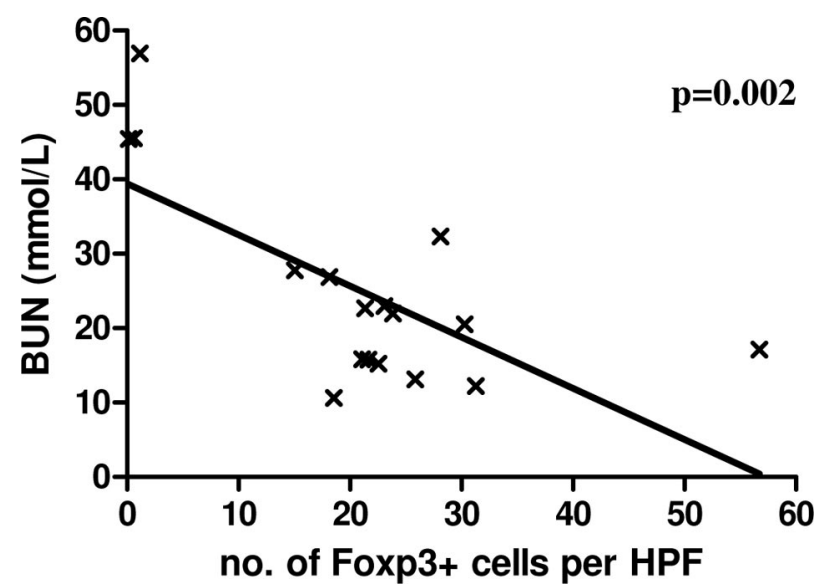

Figure 4. Correlation of renal function at time of sacrifice and Foxp3 expression in long-term $\mathrm{C} 57 \mathrm{BL} / 6$ recipients of DBA/2 kidneys. Animals with high BUN had very low numbers of Foxp $3^{+}$cells within their grafts.

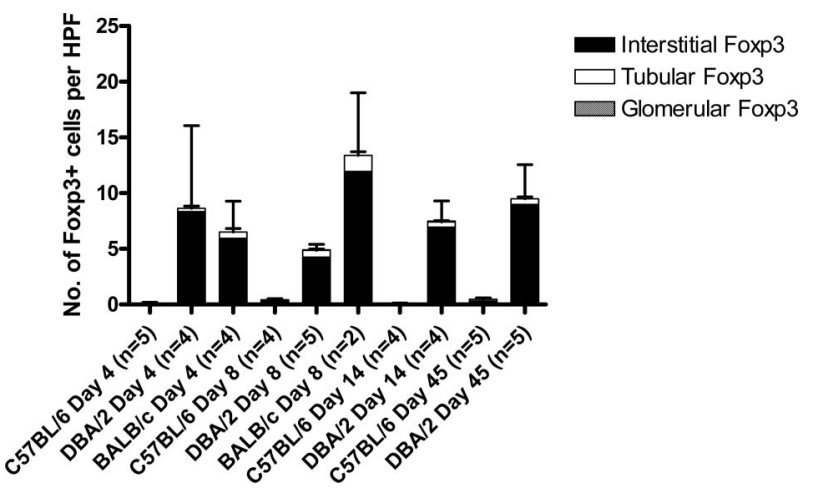

Figure 5. Localization of Foxp $3^{+}$cells within the kidney graft. The majority of positive cells are found within the interstitium, with a small proportion of all groups inside tubules and rarely in glomeruli.

particular time point are biased toward grafts that had fared better than the majority.

The less frequent glomerular Foxp ${ }^{+}$cells were found in several DBA/2 grafts, in one C57BL/6 graft, but in no BALB/c grafts. In DBA/2 kidney grafts 8 days after transplant, $4.25 \pm 1.16$ Foxp3 $^{+}$cells per HPF were found within the interstitium, with $0.61 \pm 0.12$ within tubules and $0.05 \pm 0.03$ within glomeruli (Figure 5). Importantly, even at later time points, Foxp3 ${ }^{+}$cells were still seen infiltrating tubules in DBA/2 kidney grafts $(0.5 \pm 0.18$ per HPF at 45 days), even though the BUNs were relatively normal (19.12 \pm 2.91).

\section{Higher Foxp3 $3^{+}$Cells within Recipient Spleen of Tolerant Animals}

Because Tregs are found within allografts and within secondary lymphoid organs, we examined recipient spleens to see whether a higher number of $\mathrm{Foxp3}^{+}$regulatory $T$ cells in recipients of DBA/2 kidneys could account for the better graft survival compared with recipients of BALB/c kidney grafts.

Figure 6 shows that Foxp3 ${ }^{+}$cells were found in all groups but were rare in DBA/2 heart allograft recipients (2.51 \pm 1.05 per HPF 8 days after transplant). Numbers were similar in spleens after syngeneic, DBA/2, and BALB/C kidney transplantation, until day 8 after transplant, at which point the number of $\mathrm{Foxp}^{+}$cells in spleens of BALB/c kidney recipients fell to significantly lower levels than those in DBA/2 kidney recipients

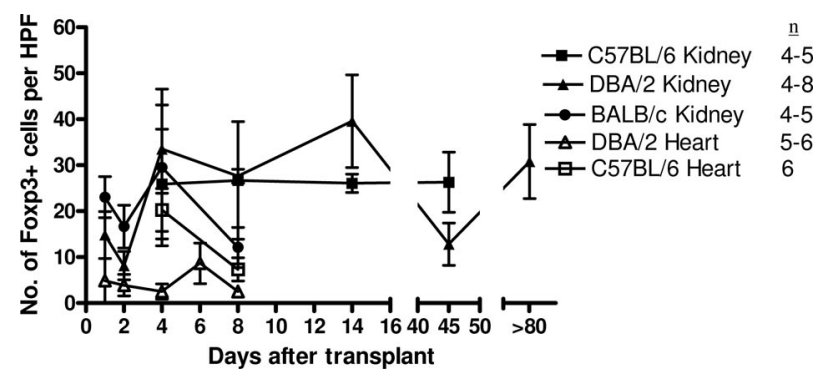

Figure 6. Foxp 3 staining in the spleens of transplant recipients. All groups had significant levels of Foxp3 expression, except recipients of DBA/2 heart allografts. 

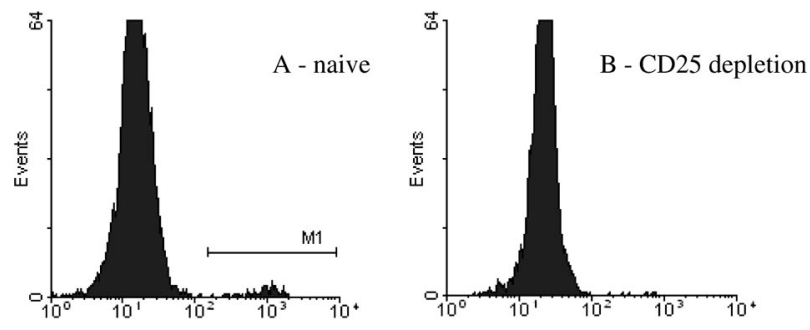

Foxp3

Figure 7. Intracellular Foxp3 staining of peripheral blood in a naïve C57BL/ 6 (A) and an anti-CD25 antibody-treated $\mathrm{C} 57 \mathrm{BL} / 6$ recipient of a DBA $/ 2$ kidney graft 5 days after transplantation $(\mathbf{B})$. Plots shown are representative. Foxp $3^{+}$ cells were almost entirely eradicated after treatment with anti-CD25 antibody.

(12.11 \pm 4.37 compared with $27.6 \pm 1.54$ per HPF, respectively, $P=0.0079)$, suggesting that Foxp3 ${ }^{+}$cells may play a role in the long-term acceptance of DBA/2 kidneys.

\section{Depletion of $\mathrm{CD}^{2} 5^{+} \mathrm{T}$ Cells in Recipients}

Although intracellular expression of Foxp3 is a reliable marker for regulatory $T$ cells in mice, to date there is no absolute cell surface marker that identifies regulatory $T$ cells. Nonetheless, regulatory T cells are highly enriched in the $\mathrm{CD}^{+}{ }^{+} \mathrm{CD}_{25}{ }^{+} \mathrm{T}$-cell compartment. Recipient mice were given four doses of depleting anti-CD25 antibody. This resulted in profound depletion of Foxp3 ${ }^{+}$cells in peripheral blood (Figure 7 ), with $0.13 \pm 0.042 \%$ of peripheral blood mononucleocytes positive for intracellular Foxp3 after treatment with anti-CD25 antibody compared with $1.2 \%$ in naïve animals, resulting in death after the second native nephrectomy, which was done 7 days after transplantation. Histological examination revealed tubulitis, but it is not possible to be certain that this is the cause of death because our data have shown that even in grafts that are accepted, there are infiltrates that mimic rejection.

\section{Discussion}

We have chosen two different mouse strains, DBA/2 and $\mathrm{BALB} / \mathrm{c}$, as kidney donors. Although they are major histocompatibility complex identical $\left(\mathrm{H}-2^{\mathrm{d}}\right)$, the majority of the former were accepted, whereas the latter were usually rejected. This allowed us to examine the infiltration of allografts by Foxp $3^{+}$Tregs in two opposing conditions and to compare them with DBA/2 heart allografts, which were always rejected. In addition, the histological diagnosis of rejection after heart transplantation is different from that of kidney allografts. The former merely requires the demonstration of infiltrating mononuclear cells, ${ }^{3}$ and the latter requires the presence of mononuclear cells infiltrating tubules. ${ }^{2}$ Furthermore, immunosuppression, which has previously been shown to inhibit the generation and function of regulatory T cells, ${ }^{18}$ is not required for long-term survival of DBA/2 allografts in this model. Therefore, another benefit of our model is that Tregs could be studied without the influence of immunosup- pressive therapies. The mechanism of tolerance in the DBA/2 donor kidney to C57BL/6 recipient has been investigated by others and is thought to be due to regulated T-cell immunity. ${ }^{17}$ In addition, CD $25^{+} \mathrm{T}$ cells (presumed to be Tregs) have been implicated in animal models of spontaneous acceptance of kidney, ${ }^{19}$ liver, ${ }^{20,21}$ and skin $^{22}$ grafts because their depletion abrogates this phenomenon.

After kidney transplantation, histological features of rejection in mice are similar to those found in human. However, these features of rejection were seen in both accepted DBA/2 and rejecting BALB/c kidneys, despite the fact that many DBA/2 kidney recipients had relatively normal $B \cup N$ at the time of sacrifice. In the clinical situation, this would be interpreted as "subclinical" rejection, defined as histological signs of rejection in the biopsy sample but with no accompanying deterioration of graft function. A proportion of these clinical cases may be due to beneficial mononuclear cells, such as regulatory $T$ cells, infiltrating the graft and mimicking rejection.

To address this possibility, we stained tissue sections for Foxp3 ${ }^{+}$cells and demonstrated their presence in both strains of kidney allograft, although they were more abundant in the tolerant DBA/2 kidney grafts at later time points. The observation that the number of Foxp3 ${ }^{+}$cells found in BALB/c grafts at day 8 is higher than DBA/2 grafts appears to be surprising at first sight. However, the number of animals in this case is small $(n=2)$, and there was no statistical difference. The reason that the number of animals in this group was small is that $83 \%$ of BALB/c kidney grafts reject acutely and $17 \%$ survive long term when transplanted into C57BL/6 recipients (Figure 1). In keeping with this observation, the majority of the BALB/c kidneys were completely infarcted or severely damaged by day 8 and so impossible to analyze. Therefore, this result is biased toward kidney grafts that have undergone less severe rejection than the majority. It is possible that these grafts would have gone on to live long term, which is seen in a small percentage of mice (Figure 1).

Even in DBA/2 kidney grafts, Foxp $3^{+}$cells were seen infiltrating tubules. Because BUN measurements were normal in these recipients at the time of sacrifice and assuming that Foxp3 ${ }^{+}$cells have a protective role, their presence within the tubules must be interpreted as mimicking rather than causing rejection. Their presence within the tubules also provides evidence of a physical route through which FOXP3 mRNA could be detected in urine of patients after transplantation. ${ }^{12}$

It is interesting to note that Foxp $3^{+}$cells were rarely seen in syngeneic kidney and heart grafts, in keeping with the notion that an immune response is necessary to generate regulatory $T$ cells, although this does not offer definitive proof. It is not possible to determine the relative importance of graft infiltrating $\mathrm{Foxp}^{+} \mathrm{T}$ cells compared with splenic Foxp3 ${ }^{+} \mathrm{T}$ cells from the experiments described here, but the higher numbers of Foxp $^{+} \mathrm{T}$ cells within the spleens of DBA/2 kidney recipients is compatible with the notion that splenic 
Tregs have a stronger influence on the graft outcome than intragraft Tregs.

Some recipients of DBA/2 kidney allografts demonstrated a rise in BUN over time. It is unclear why certain recipients accepted their grafts indefinitely whereas others did not. Biological variation, previous immune history of the recipient, concomitant infection, or varying degrees of surgical trauma and ischemic reperfusion injury at the time of transplant may all play a role. The observation that recipients with higher BUN measurements had lower numbers of $\mathrm{Foxp}^{+}$cells within their grafts is highly suggestive, but not definitive proof, that the long-term acceptance of DBA/2 kidney grafts is an active process, requiring the continuing presence of regulatory $T$ cells to suppress alloreactivity. In animals with low Foxp3 ${ }^{+}$cell counts within the transplanted kidneys, typical features of chronic allograft nephropathy were seen, compared with grafts from animals with normal BUN that showed well-preserved tubules. Although difficult to quantify objectively, multiple clusters of mononuclear cells were noted visually as typical features in these grafts with normal BUN. The observation that Foxp ${ }^{+}$cells are among these clusters (Figure 2I) provides a physical basis for cell-to-cell contact between Tregs and alloreactive $\mathrm{T}$ cells.

Because Foxp3 is an intracellular transcription factor and not expressed on the cell surface, depleting Foxp3 ${ }^{+}$ cells cannot be readily achieved. Because $\mathrm{Foxp}^{+}$cells are highly enriched in $\mathrm{CD} 4^{+} \mathrm{CD} 25^{+} \mathrm{T}$ cells, other investigators have used monoclonal antibodies against CD25 and shown that tolerance was abrogated. ${ }^{19-22}$ However, CD25 is also expressed on activated T cells, and therefore, failure to abrogate tolerance does not imply that Foxp3 ${ }^{+} \mathrm{T}$ cells are not involved. To investigate this in our model, we treated recipient mice with rat anti-mouse CD25 monoclonal antibodies. This resulted in death after the second native nephrectomy. Postmortem examination showed signs of rejection. However, because we have already demonstrated that tubulitis could be seen in tolerant DBA/2 donor grafts, the cause of death cannot be attributed to rejection with certainty. Furthermore, the course of antibody treatment was intense, with one intravenous and three intraperitoneal injections of xeno antibodies. A high proportion of recipients died between days 3 and 6 after transplantation, before second nephrectomy, and had to be excluded from the experiment. This is most likely due to side effects of repeated injections of xeno antibodies, which can elicit a serum sickness-like immune response. A less-intense course of antibody treatment, while at the same time ensuring that Foxp3 ${ }^{+} \mathrm{T}$ cells are adequately depleted, may help to resolve this.

Translating conclusions drawn from animal experiments to the clinical situation should always be done with caution, recognizing the limitations. In humans, spontaneous acceptance of kidney allografts is rare but does happen and has been seen in between 16 and 19\% of kidney recipients who discontinued all immunosuppressive therapy against medical advice. ${ }^{23,24}$ Furthermore, many patients enjoy prolonged survival of their kidney allografts on minimal immunosuppression, suggesting that various degrees of "tolerance" can exist. The observations presented here may have an important bearing in the clinical situation, especially on this group of patients. Our data suggest that infiltration of tubules by mononuclear cells may not be, in contrast to current opinion, an absolute marker of rejection, because it also occurs in tolerant kidneys. It may be of use to analyze this infiltrate more closely and to identify any regulatory element that may be incorporated within it.

\section{References}

1. Al-Awwa IA, Hariharan S, First, MR: Importance of allograft biopsy in renal transplant recipients: correlation between clinical and histological diagnosis. Am J Kidney Dis 1998, 31(Suppl 1):S15-S18

2. Racusen LC, Solez K, Colvin RB, Bonsib SM, Castro M-C, Cavallo T, Croker BP, Demetris AJ, Drachenberg CB, Fogo AB, Furness P, Gaber LW, Gibson IW, Glotz D, Goldberg JC, Grande J, Halloran PF, Hansen HE, Hartley B, Hayry PJ, Hill CM, Hoffman EO, Hunsicker LG, Lindblad A, Marcussen N, Mihatsch MJ, Nadasdy T, Nickerson P, Olsen TS, Papadimitriou JC, Randhawa PS, Rayner DC, Roberts I, Rose S, Rush D, Salinas-Madrigal L, Salomon DR, Sund S, Taskinen E, Trpkov K, Yamaguchi Y: The Banff 97 working classification of renal allograft pathology. Kidney Int 1999, 55:713-723

3. Stewart S, Winters GL, Fishbein MC, Tazelaar HD, Kobashigawa J, Abrams J, Burke MM, Demetris AJ, Hammond E, Itescu S, Marboe CC, McManus B, Reed EF, Reinsmoen NL, Rodriguez ER, Rose AG, Rose M, Suciu-Focia N, Zeevi A, Billingham ME: Revision of the 1990 working formulation for the standardization of nomenclature in the diagnosis of heart rejection. J Heart Lung Transplant 2005, 24:1710-1720

4. Wood KJ, Sakaguchi S: Regulatory T cells in transplantation tolerance. Nat Rev Immunol 2003, 3:199-210

5. Fontenot JD, Gavin MA, Rudensky AY: Foxp3 programs the development and function of CD4+CD25+ regulatory $T$ cells. Nat Immunol 2003, 4:330-336

6. Hori S, Nomura T, Sakaguchi S: Control of regulatory T cell development by the transcription factor Foxp3. Science 2003, 299:1057-1061

7. Khattri R, Cox T, Yasayko S, Ramsdell F: An essential role for Scurfin in CD4+CD25+ T regulatory cells. Nat Immunol 2003, 4:337-342

8. Qin S, Cobbold SP, Pope H, Elliot J, Kioussis D, Davies J, Waldmann H: "Infectious" transplantation tolerance. Science 1993, 259:974-977

9. Ochando JC, Yopp AC, Yang Y, Garin A, Li Y, Boros P, Llodra J, Ding $Y$, Lira SA, Krieger NR, Bromberg JS: Lymph node occupancy is required for the peripheral development of alloantigen-specific Foxp3+ regulatory T cells. J Immunol 2005, 174:6993-7005

10. Graca L, Cobbold SP, Waldmann H: Identification of regulatory T cells in tolerated allografts. J Exp Med 2002, 195:1641-1646

11. Zheng XX, Sanchez-Fueyo A, Sho M, Domenig C, Sayegh MH, Strom TB: Favorably tipping the balance between cytopathic and regulatory $T$ cells to create transplantation tolerance. Immunity 2003, 19:503-514

12. Muthukumar T, Dadhania D, Ding R, Snopkowski C, Naqvi T, Lee JB, Hartono C, Li B, Sharma VK, Seshan SV, Kapur S, Hancock WW, Schwartz JE, Suthanthiran M: Messenger RNA for FOXP3 in the urine of renal allograft recipients. N Engl J Med 2005, 353:2342-2345

13. Han WR, Murray-Segal LJ, Mottram PL: Modified technique for kidney transplantation in mice. Microsurgery 1999, 19:272-274

14. Corry RJ, Winn HJ, Russell PS: Primarily vascularized allografts of hearts in mice. Transplantation 1973, 16:343-350

15. Billingham RE, Medawar PB: The technique of free skin grafting in mammals. J Exp Biol 1951, 28:385

16. McManus JFA: Histological and histochemical uses of periodic acid. Stain Technol 1948, 23:99-108

17. Bickerstaff AA, Wang J-J, Pelletier RP, Orosz CG: Murine renal allografts: spontaneous acceptance is associated with regulated $\mathrm{T}$ cell-mediated immunity. J Immunol 2001, 167:4821-4827

18. Wang $H$, Zhao L, Sun Z, Sun L, Zhang B, Zhao Y: A potential side 
effect of cyclosporin A: inhibition of CD4+CD25+ regulatory T cells in mice. Transplantation 2006, 82:1484-1492

19. Wang C, Cordoba S, Bertolino P, Alexander S, Wu M, Allen R, McCaughan G, Bishop G: Examination of the immune mechanism of spontaneous tolerance of renal allografts in a mouse model. Immunol Cell Biol 2006, 84:A29-A30

20. Jiang X, Morita M, Sugioka A, Harada M, Kojo S, Wakao H, Watarai H, Ohkohchi N, Taniguchi M, Seino K: The importance of CD25+CD4 ${ }^{+}$ regulatory $T$ cells in mouse hepatic allograft tolerance. Liver Transplant 2006, 12:1112-1118

21. Li W, Carper K, Liang Y, Zheng XX, Kuhr CS, Reyes JD, Perkins DL, Thomson AW, Perkins JD: Anti-CD25 mAb administration prevents spontaneous liver transplant tolerance. Transplant Proc 2006, 38:3207-3208

22. Benghiat FS, Graca L, Braun MY, Detienne S, Moore F, Buonocore S, Flamand V, Waldmann H, Goldman M, Le Moine A: Critical influence of natural regulatory $\mathrm{CD} 25^{+} \mathrm{T}$ cells on the fate of allografts in the absence of immunosuppression. Transplantation 2005, 79:648-654

23. Uehling DT, Hussey JL, Weinstein AB, Wank R, Bach FH: Cessation of immunosuppression after renal transplantation. Surgery 1976, 79:278-282

24. Zoller KM, Cho SI, Cohen JJ, Harrington JT: Cessation of immunosuppressive therapy after successful transplantation: a national survey. Kidney Int 1980, 18:110-114 